\title{
Towards a Deterministic High-Frequency Shallow Water Ray Propagation Model
}

\author{
L. Pautet* and E. Pouliquen* \\ ${ }^{*}$ NATO Undersea Research Centre, viale San Bartolomeo 400, 19038 La Spezia, Italy
}

\begin{abstract}
High frequency acoustic scattering from the ocean bottom and the ocean surface has been the subject of continuing interest for many years. Data and models have shown that as the signal frequency increases, the scattering pattern from such rough surfaces evolves from specular to quasi omni-directional. Multipath structures observed in shallow waters exhibit angular spreads that cannot be explained by assuming purely specular reflection. Most ray propagation models treat surface bounces as being essentially specular which poorly accounts for the multipath structures and in turn introduce errors in the estimation of the propagated and reverberated fields. For several applications such as high frequency Synthetic Aperture Sonar (SAS), minehunting and communications there is a need for reliable and fast propagation models able to correctly treat scattering at the rough waveguide boundaries. The Bellhop ray tracing model has been modified to introduce deterministic properties of the sea surface and sea bottom. Scattering from these surfaces is treated using solutions from the integral equation. Simulations display a significant effect of the angular spreading of the multipath structure increasing with roughness that is closer to the one observed on in-situ data.
\end{abstract}

\section{INTRODUCTION}

Applications such as communications, minehunting, high-frequency SAS have brought the focus on ocean acoustics modeling to shallow waters from tens to hundreds of metres and high-frequencies in the tens of $\mathrm{kHz}$. To be accurate in these conditions, sound propagation modeling needs to take into account scattering occuring at the multiple interactions with the rough boundaries.

High frequency scattering from rough surfaces has been the object of many studies. Scattering models span from the bidimensional "exact" solution obtained with the integral equation [1] to bi- and tri-dimensional Kirchhoff models [1], small perturbation methods [2], composite-roughness models [3], small slope approximations [4, 5, 6], etc. The limits and the domain of validity of the different models and approximations have been investigated through extensive numerical work. However, only a few propagation models have included the effect of scattering by rough boundaries. The most basic approach has been to associate an angle-dependent surface loss to the specular direction discarding the fact that energy is also scattered in non-specular directions [7]. Some sophisticated parabolic equation models are able to take into account the whole scattered field by discretizing the surface in order to capture the large scale scattering of the rough boundaries $[8,9]$. However, at high frequency, this approach becomes computationally challenging due to the required spatial discretization. There are also some questions about the accuracy of a stair-case discretization of a surface with a multi-scale 
roughness spectrum when the size of the wavelength is of the same order as the size of the RMS-roughness. Normal modes have also been used to calculate scattered field by combining a normal mode decomposition with a module describing the intermodal transfer of energy due to scattering [10]. Again, at high frequencies, the increasing number of modes makes this approach non suitable. In order to find a compromise between computational time and accuracy, a ray tracing approach is presented here.

High frequency scattering by a rough surface observed experimentally in a very shallow water environment shows that a significant portion of the energy is scattered in directions other than the specular direction [11, 12]. This calls into question the use of ray theory based on Snell's law to model propagation. Similar problems are encountered in the EM field. In atmospheric science, it can be solved by using a Monte Carlo propagation model to simulate the photons paths within the ocean-atmosphere system as they are scattered by particules and the air/sea interface [13, 14]. This paper presents an approach similar to the one used in the EM field applied to a commonly used ray propagation model, Bellhop [15]. A statistical scattering kernel based on the integral equation is combined with this ray tracer hereafter referred to as the "Bellodds" model. The implementation of the scattering kernel is described before presenting results from a few simulations.

\section{SCATTERING KERNEL}

The Bellhop ray tracing propagation model is modified by adding an incoherent scattering component. The interface patch on which the ray impinges can be considered as a small antenna, which creates a scattering beam pattern as illustrated in Fig. 1. Each ray impinging on a rough boundary with an incident angle $\theta_{i}$ is scattered in a direction $\theta_{s}$, determined statistically as a function of the local scattering strength. The idea is that if enough rays are launched, the resulting acoustic field becomes statistically meaningful. Interface bistatic scattering coefficients are calculated for a series of generated rough profiles with chosen spatial statistics (e.g. Gaussian, power-law, Pierson-Moskowitz spectra) using the integral equation [1]. The scattering coefficient is treated as $P_{\theta_{i}}\left(\theta_{s}\right)$, the probability for a ray with an incident angle $\theta_{i}$ to be "reflected" in the direction given by the scattered angle $\theta_{s}$ :

$$
P_{\theta_{i}}\left(\theta_{s}\right)=\frac{m\left(\theta_{i}, \theta_{s}\right)}{\int_{\alpha=-\pi / 2}^{\pi / 2} m\left(\theta_{i}, \alpha\right) d \alpha},
$$

where $\sigma\left(\theta_{i}, \theta_{s}\right)=10 \log \left(m\left(\theta_{i}, \theta_{s}\right)\right)$ is the scattering strength. The principle of energy conservation is satisfied by an appropriate normalization but a larger number of rays need to be sent in order to obtain a statiscally meaningful acoustic field in the waveguide. Fig. 2 and Fig. 3 show how the averaged scattering strength and corresponding probabilities of scattering evolve with increasing surface roughness at a given frequency. The probability for the energy to be non-specular (i.e. incoherent) is significant and increases with roughness and/or frequency. 


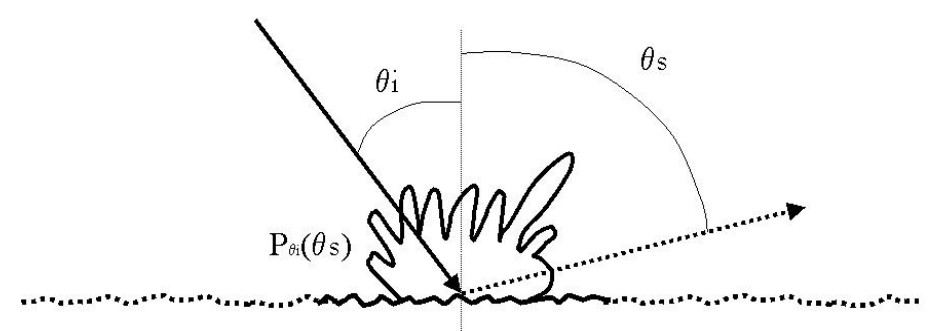

FIGURE 1. Schematic of the ray scattering process showing the scattering beam pattern in terms of probability and a ray being scattered at a non-specular angle.

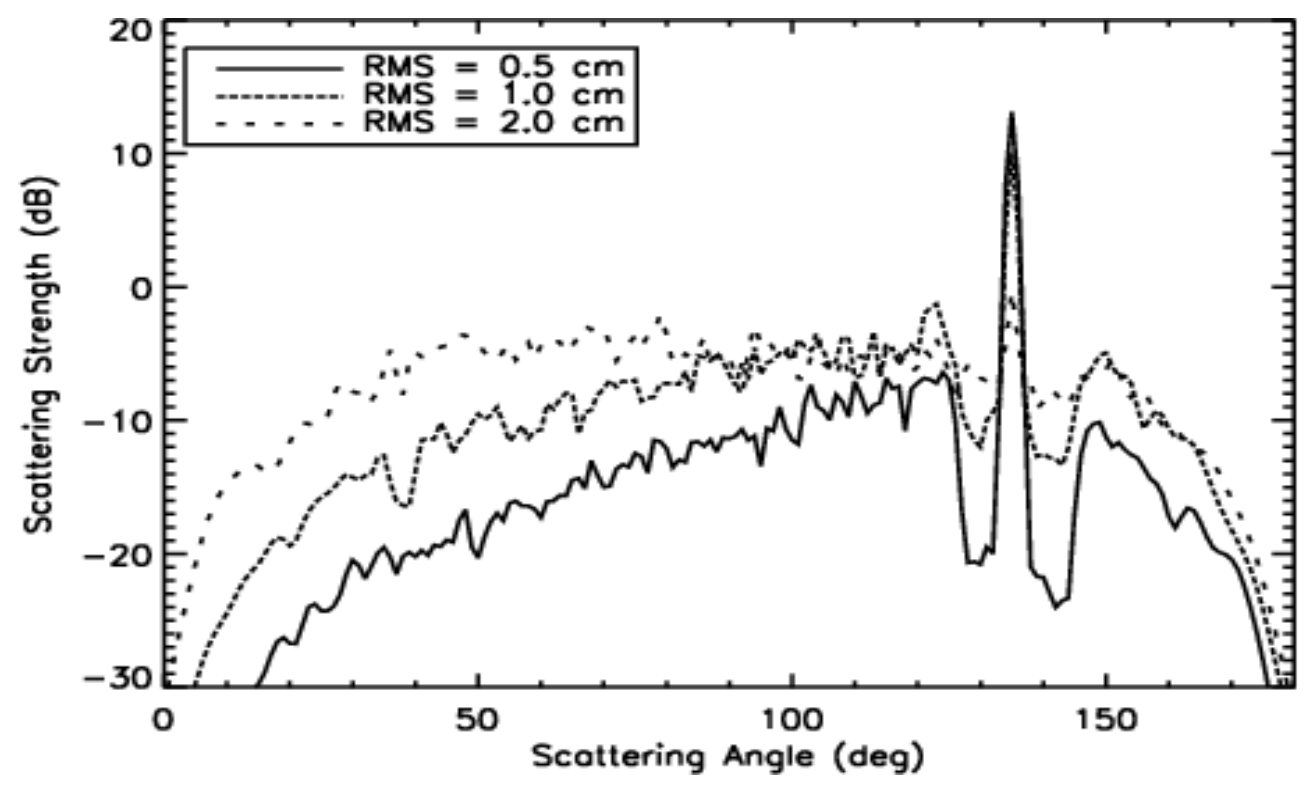

FIGURE 2. Scattering strength at $20 \mathrm{kHz}$ from a surface having a saturated power-law spectrum $\left(\gamma=3.6, k_{s}=30 \mathrm{rad} / \mathrm{m}\right)$ computed using the integral equation [1]. Results are averaged over 50 surfaces and correspond to $45^{\circ}$ incident angle.

\section{RESULTS}

With the use of a pre-calculated lookup table containing a series of scattering probabilities depending on the incident and scattered angles and various surface roughness 


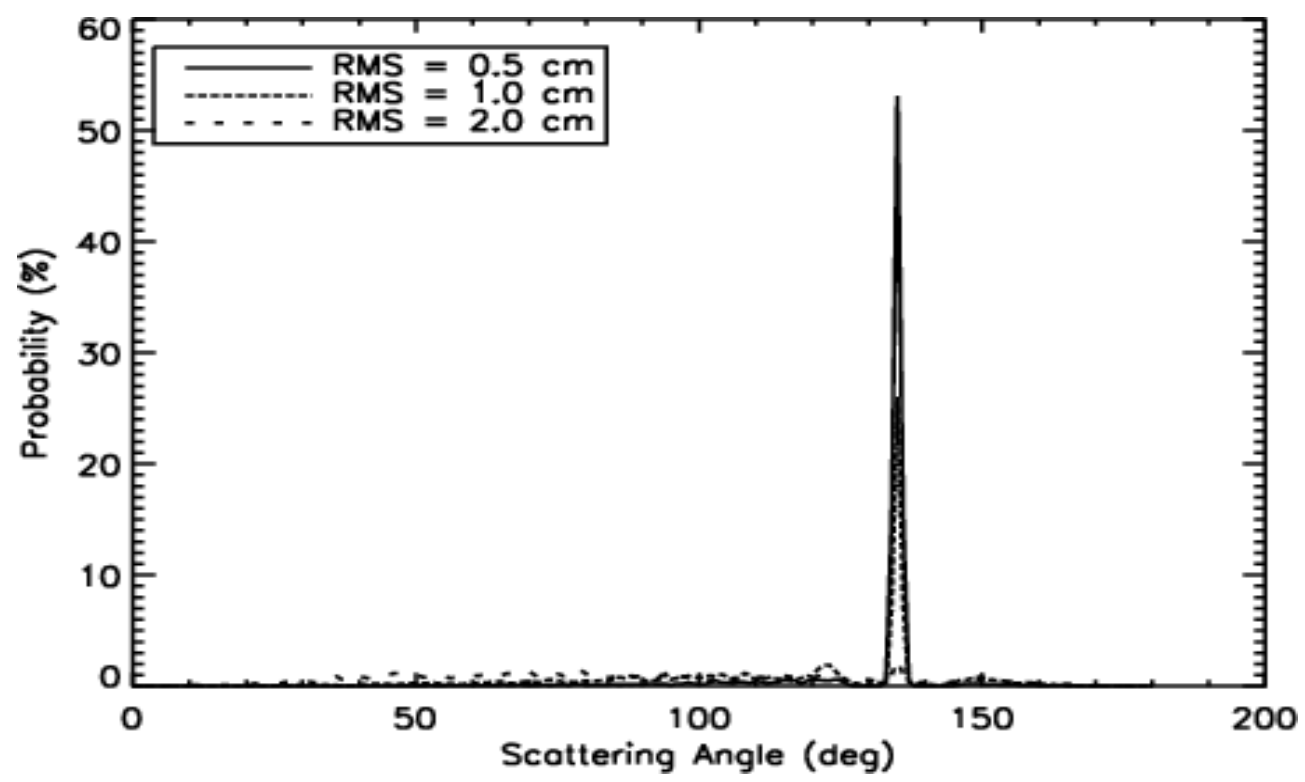

FIGURE 3. Scattering probabilities extracted from Fig. 2. The probability for the energy to be scattered in the exact specular direction is $55 \%$ for the smoothest surface, $27 \%$ for the intermediate surface, and less than $5 \%$ for the roughest surface.

properties, Bellodds can be used to simulate high frequency propagation and reverberation. Fig. 4 shows the results obtained in a monostatic case. A $100 \mathrm{kHz}$ source is placed in the middle of a $20 \mathrm{~m}$ deep channel and transmits like a sidescan sonar towards the seabed. The bottom interface is generated using a saturated power-law spectrum $\left(\gamma=3.6, k_{s}=30\right.$ $\mathrm{rad} / \mathrm{m}$ ) with a RMS-roughness of $1 \mathrm{~cm}$. The sea surface is considered to be quasi-flat with a RMS-roughness of $1 \mathrm{~mm}$. The clearly visible paths are the bottom path (B path), the bottom/surface path (BS path) and the bottom/surface/bottom path (BSB path). Each of them displays an angular spreading caused by the seabed roughness. There are two competing effects: an increase of the angular spread with the number of surface bounces and the classical attenuation loss. This explains why the bottom/surface/bottom shows the strongest angular spread in this case. Similar behavior was observed in data acquired in situ [11].

As the roughness increases, the scattering probability $P_{\theta_{i}}\left(\theta_{s}\right)$ becomes almost uniform (Fig. 3). In fact, the probability could be schematized as being uniform for all scattered angles except for the specular angle. Equation 1 can be simplified to the following expression.

$$
P_{\theta_{i}}\left(\theta_{S}\right)=1-P_{\text {spec }}+\delta_{\theta_{\text {spec }}}\left(\theta_{s}\right)\left[2 P_{\text {spec }}-1\right],
$$

where $P_{\text {spec }}$ is the probability for the scattered ray to be a specular ray, $\theta_{\text {spec }}$ is the specular angle, and $\delta_{\theta_{\text {spec }}}\left(\theta_{S}\right)$ is the Kronecker delta symbol.

An estimate of waveguide properties can then be calculated with only the specular probability $p_{\text {spec }}$ as roughness input. Fig. 5 and Fig. 6 illustrate transmission loss results obtained using this approach. The channel used is a isovelocity channel, $20 \mathrm{~m}$ deep with a source placed at $10 \mathrm{~m}$ depth over a silt bottom. The calculations are made first without 


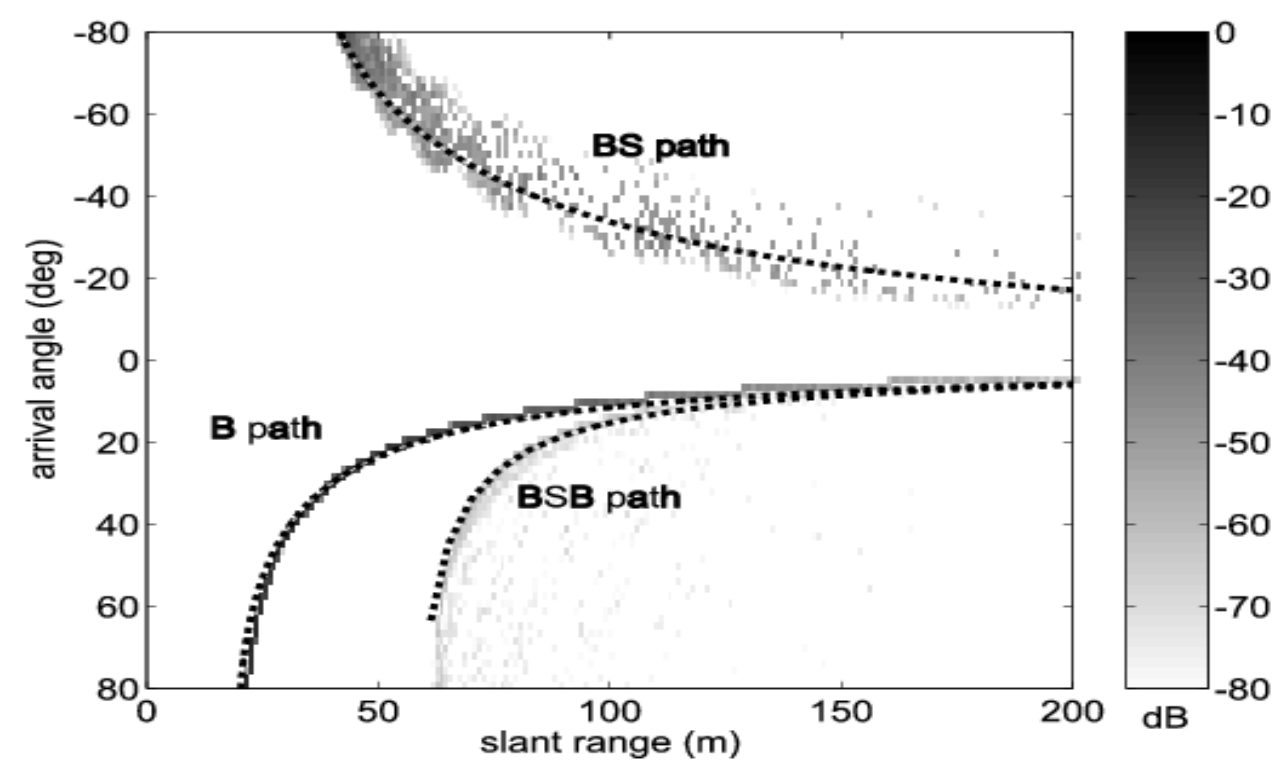

FIGURE 4. Estimate of reverberation versus vertical arrival angle and slant range for a flat sea surface and a bottom RMS roughness of $1 \mathrm{~cm}$ at $100 \mathrm{kHz}$. The lines represent the theoretical specular paths.

any roughness, and then with a 90 percent probability of having a specular reflection at each boundary, the rest of the rays being uniformly scattered in the other directions. As the roughness increases more energy is dissipated in the first bounces: rays launched at angles that would be above the critical angle may be scattered at angles below the critical angle and be absorbed by the bottom [16]. As expected, the multipath structure gets more and more disturbed as range increases. The refocusing of the fiel observed is coherent with a isovelocity scenario with source at mid depth. Any changes in sound speed profile or source depth would eliminate this regular focusing. The interest of using such a particular scenario is to illustrate the decay of the structure as the roughness increases, i.e. as scattering inhibits the perfect refocusing.

\section{CONCLUSION}

Through a statistical description of the scattering process, a deterministic ray-based model can be used to simulate the propagation or reverberation in a rough waveguide. As observed in data, simulations show an angular spreading of the multipath structure in reverberation and an increased loss in the propagation. In situ, water column instabilities and small source/receivers movements would have a similar impact on multipath structure and transmission loss. Unfortunately, it is impossible to separate these effects especially at these high frequencies. However boundary roughness appears to be a significant factor in channel coherence at high frequency. One advantage of Bellodds is that because of its statistical nature, each run is different from the next one, so the effects of even these small fluctuations can be simulated using a similar approach.

Future work will address volume scattering within the water column and the sediment. 


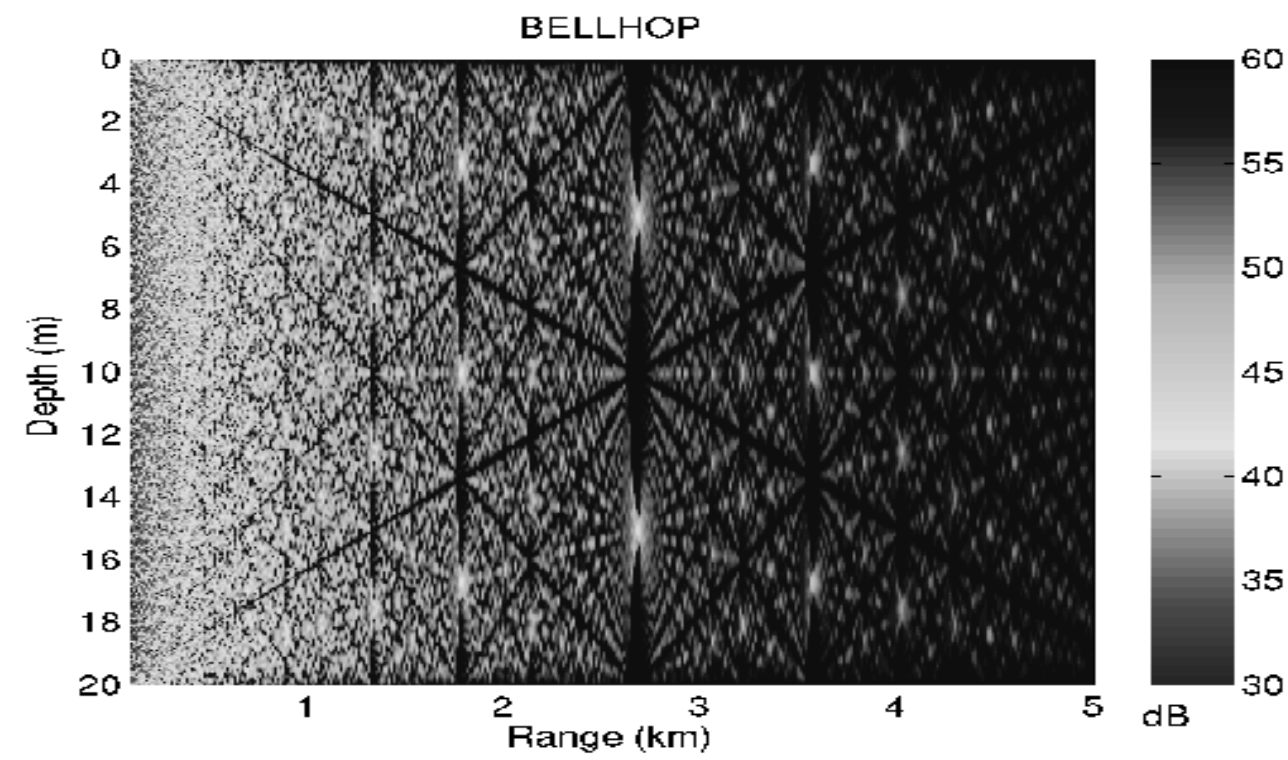

FIGURE 5. Transmission loss for a $20 \mathrm{kHz}$ source placed at $10 \mathrm{~m}$ depth in a $20 \mathrm{~m}$ wave guide with a flat silty bottom and sea surface.

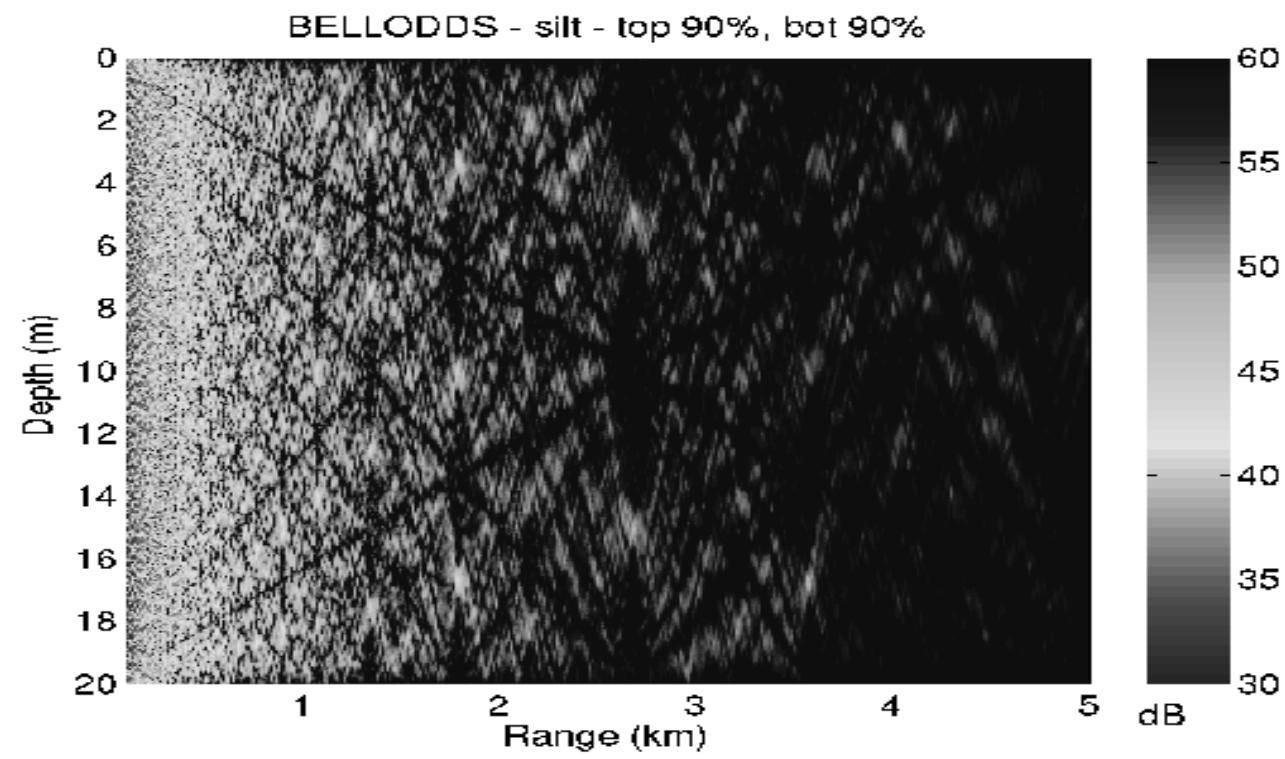

FIGURE 6. Transmission loss for a $20 \mathrm{kHz}$ source placed at $10 \mathrm{~m}$ depth in a $20 \mathrm{~m}$ wave guide with a slightly rough silty bottom and sea surface (90 percent of the energy is reflected in the specular direction).

\section{REFERENCES}

1. Thorsos, E., Journal of the Acoustical Society of America, 83, 78-92 (1988).

2. Thorsos, E., and Jackson, D., Journal of the Acoustical Society of America, 86, 261-277 (1989).

3. Daniel, S. M., and Gorman, A., Journal of the Acoustical Society of America, 73, 1476-1486 (1983).

4. Thorsos, E., and Broschat, S., Journal of the Acoustical Society of America, 97, 2082-2093 (1995). 
5. Broschat, S., and Thorsos, E., Journal of the Acoustical Society of America, 101, 2615-2625 (1997).

6. Yang, T., and Broschat, S., IEEE Transactions on Antennas and Propagation, 40, 1505-1512 (1992).

7. Moore-Head, M., and Jobst, W., Journal of the Acoustical Society of America, 86, 247-251 (1989).

8. Purrington, R., Journal of Computational Acoustics, 2, 147-160 (1994).

9. Rosenberg, A., Journal of the Acoustical Society of America, 105, 144-153 (1999).

10. Ellis, D., Journal of the Acoustical Society of America, 97, 2804-2814 (1995).

11. Wang, L., Davies, G., Bellettini, A., and Pinto, M., "Multipath effect on DPCA micronavigation of a synthetic aperture sonar," in Impact of Littoral Envionmental Variability on Acoustic Predictions and Sonar Performance, 2002, pp. 465-472.

12. Dahl, P., IEEE Journal of Oceanic Engineering, 26, 141-151 (2001).

13. Breon, F., Journal of Atmospheric Science, 49, 1221-1232 (1992).

14. Frouin, R., Pouliquen, E., and Breon, F., "Ocean color remote sensing using polarization properties of reflected sunlight," in Proceedings of the 6th international conference on Physical Measurements and Signatures in Remote Sensing, 1994.

15. Porter, M., and Liu, Y., Theoretical and Computational Acoustics, 2, 947-956 (1994).

16. Thorsos, E., Henyey, F., Williams, K., Elam, W., and Reynolds, S., "Simulation of temporal and spatial variability in shallow water propagation," in Impact of Littoral Envionmental Variability on Acoustic Predictions and Sonar Performance, 2002, pp. 337-344. 